\title{
On the relation between dissipativity and discounted dissipativity
}

\author{
Matthias A. Müller and Lars Grüne
}

\begin{abstract}
In this paper, we consider a recently introduced notion of discounted strict dissipativity which is of interest, e.g., in the context of discounted optimal control and economic model predictive control. We consider the question whether (standard) strict dissipativity implies discounted strict dissipativity for discounting factors close enough to one. While in general, this need not be the case, we show that it is indeed true under certain regularity conditions.
\end{abstract}

\section{INTRODUCTION}

Economic model predictive control (MPC) is an MPC approach where the primary control objective is not the stabilization of some (given) setpoint, but the optimization of some general performance criterion. This leads to the fact that the repeatedly solved optimal control problem uses a stage cost function which is not necessarily positive definite with respect to a given setpoint [1]. In recent years, economic MPC has received significant attention, and various different economic MPC schemes have been proposed and analyzed in the literature, see, e.g., the survey articles [2,3].

In the study of economic MPC schemes, a certain dissipativity property has turned out to play a crucial role [4]. The concept of dissipativity has been introduced by Willems [5] and has since then found many different applications in systems and control theory. In the context of economic MPC, a certain dissipativity condition was used as an (almost) equivalent characterization of steady-state optimality $[1,6]$, in order to classify turnpike properties of the underlying optimal control problem [7-9], and in order to establish closed-loop convergence and performance guarantees $[1,7$, $10,11]$.

In recent works $[12,13]$, some of the above results were extended to a setting where a discounted stage cost is used. Such discounted optimal control problems are, e.g., widely used in economics, where discounting is used in order to account for the fact that economic utility in the far future is typically weighed less than that of the near future, see, e.g., [14]. Recent applications of this setting include models for climate change economics such as the DICE model (Dynamic Integrated model of Climate and the Economy, [15]), which has also been analyzed using economic MPC approaches, see [16]. In order to establish similar results as in the undiscounted case, a suitable notion of discounted

Matthias A. Müller is with the Institute for Systems Theory and Automatic Control, University of Stuttgart, 70550 Stuttgart, Germany. His work was supported by DFG Grant MU3929/1-1 and by the Eliteprogramme for Postdocs of the Baden-Württemberg Foundation. matthias.muellerdist.uni-stuttgart.de

Lars Grüne is with the Mathematical Institute, University of Bayreuth, 95440 Bayreuth, Germany. His work was supported by DFG Grant GR1569/13-1 lars.grueneduni-bayreuth. de strict dissipativity was introduced in [12], and it was shown that again statements about steady-state optimality and the behavior of optimal solutions to discounted optimal control problems can be made using a specific discounted (strict) dissipativity condition.

Motivated by the above, in this paper we study the relation between standard (undiscounted) strict dissipativity and discounted strict dissipativity. Formally, the discounted strict dissipativity condition from [12] becomes the well known undiscounted condition with the discount rate is set to $\beta=1$. This naturally leads to the so far unanswered question, whether strict dissipativity implies discounted strict dissipativity for discount factors close enough to one, which we investigate in this paper. If the answer was positive, this would imply that steady-state optimality, turnpike properties and closed-loop performance and convergence statements in economic MPC are robust with respect to "mild enough" discounting. While in general, this need not be the case, we show that it is indeed true under certain regularity conditions known from nonlinear programming.

The remainder of the paper is structured as follows. Section II gives preliminaries on notation and the considered problem setup; furthermore, we briefly review the role of dissipativity in economic MPC. Section III presents the main results showing under what conditions strict dissipativity implies discounted strict dissipativity for discount factors close enough to one. This is first done for the specific supply rate which is of interest in (discounted) optimal control and economic MPC in Section III-A. After that, Section III-B shows that the results can be extended to general supply rates and can hence also be of interest in a different context. In Section IV, we illustrate the obtained results with a simple example, before we conclude the paper in Section V.

\section{PRELIMINARIES AND SETUP}

\section{A. Notation and system class}

For a continuously differentiable function $F(x), F$ : $\mathbb{R}^{n} \rightarrow \mathbb{R}^{m}$, denote by $\nabla_{x} F(\bar{x}) \in \mathbb{R}^{m \times n}$ the Jacobian matrix of $F$, evaluated at the point $\bar{x}$. For a twice continuously differentiable function $F(x), F: \mathbb{R}^{n} \rightarrow \mathbb{R}$, denote by $\nabla_{x}^{2} F(\bar{x}) \in \mathbb{R}^{n \times n}$ the Hessian matrix of $F$, evaluated at the point $\bar{x}$. For a vector $a=\left[\begin{array}{lll}a_{1} & \ldots & a_{n}\end{array}\right]^{T} \in \mathbb{R}^{n}$, denote by $\operatorname{diag}(a) \in \mathbb{R}^{n \times n}$ a diagonal matrix with diagonal entries $a_{i}$.

We consider nonlinear discrete-time systems of the form

$$
x(k+1)=f(x(k), u(k)), \quad x(0)=x_{0}
$$

for a function $f: \mathbb{R}^{n} \times \mathbb{R}^{m} \rightarrow \mathbb{R}^{n}$. System (1) is subject to state and input constraints $(x, u) \in \mathbb{Y}$ for some compact 
set $\mathbb{Y} \subseteq \mathbb{R}^{n} \times \mathbb{R}^{m}$, and we define $\mathbb{X}:=\left\{x \in \mathbb{R}^{n}\right.$ : $\exists u \in \mathbb{R}^{m}$ s.t. $\left.(x, u) \in \mathbb{Y}\right\}$ and $\mathbb{U}:=\left\{u \in \mathbb{R}^{m}: \exists x \in\right.$ $\mathbb{R}^{n}$ s.t. $\left.(x, u) \in \mathbb{Y}\right\}$. For a given control sequence $u=$ $(u(0), \ldots, u(K)) \in \mathbb{U}^{K+1}$ (or $\left.u=(u(0), \ldots) \in \mathbb{U}^{\infty}\right)$ and initial condition $x_{0}$, the corresponding solution of system (1) is denoted by $x_{u}\left(k, x_{0}\right)$ or just by $x(k)$ if there is no ambiguity about $x_{0}$ and $u$. For a given $x_{0} \in \mathbb{X}$, the set of all feasible control sequences of length $N$ is denoted by $\mathbb{U}^{N}\left(x_{0}\right)$, where a feasible control sequence is such that $(x(k), u(k)) \in \mathbb{Y}$ for all $k=0, \ldots, N-1$ and $x(N) \in \mathbb{X}$. Similarly, the set of all feasible control sequences of infinite length is denoted by $\mathbb{U}^{\infty}\left(x_{0}\right)$. In the following, we assume for simplicity that $\mathbb{U}^{\infty}\left(x_{0}\right) \neq \emptyset$ for all $x_{0} \in \mathbb{X}$, i.e., the set $\mathbb{X}$ is controlled invariant. We expect that for the following results, this assumption can be relaxed by restricting initial values to the viability kernel $\mathbb{X}_{\infty}:=\left\{x_{0} \in \mathbb{X}: \mathbb{U}^{\infty} \neq \emptyset\right\}$; however, the technical details of this extension are beyond the scope of this paper. Finally, a point $\left(x^{e}, u^{e}\right) \in \mathbb{Y}$ is called an equilibrium of system (1) if $x^{e}=f\left(x^{e}, u^{e}\right)$.

\section{B. Dissipativity and economic MPC}

We now briefly review the concepts of dissipativity and discounted dissipativity and discuss their role in economic MPC. The concept of dissipativity dates back to Willems [5] (see also [17] for a discrete time version) and is as follows.

Definition 1: Given an equilibrium $\left(x^{e}, u^{e}\right)$, the system (1) is strictly dissipative with respect to the supply rate $s: \mathbb{Y} \rightarrow \mathbb{R}$ if there exists a storage function $\lambda: \mathbb{R}^{n} \rightarrow \mathbb{R}$ bounded from below and a function $\alpha \in \mathcal{K}_{\infty}$ such that the following inequality is satisfied for all $(x, u) \in \mathbb{Y}$ :

$$
\lambda(f(x, u))-\lambda(x) \leq s(x, u)-\alpha\left(\left|\left(x-x^{e}, u-u^{e}\right)\right|\right) .
$$

Remark 2: If (2) is satisfied with $\alpha \equiv 0$, then system (1) is called dissipative. Furthermore, we remark that the standard definition of strict dissipativity uses $\alpha\left(\left|x-x^{e}\right|\right)$ instead of $\alpha\left(\left|\left(x-x^{e}, u-u^{e}\right)\right|\right)$ in (2). The results in Section III require this slightly stronger definition, which in the context of economic MPC has recently also been used, e.g., in [6, $18,19]$.

In recent years, a certain (strict) dissipativity property has turned out to play a crucial role in the context of economic MPC, both for classifying optimal operating behaviors as well as for closed-loop performance and convergence statements. In economic MPC, at each time $t \in \mathbb{N}_{0}$ with measured system state $x:=x(t)$, the following finite-horizon optimal control problem is solved:

$$
\min _{u \in \mathbb{U}^{N}(x)} \hat{J}_{N}(x, u) \text { with } \hat{J}_{N}(x, u)=\sum_{k=0}^{N-1} \ell\left(x_{u}(k, x), u(k)\right),
$$

where $\ell: \mathbb{R}^{n} \times \mathbb{R}^{m} \rightarrow \mathbb{R}$ is some general (not necessarily positive definite) stage cost function. Then, given an optimal solution $u^{0} \in \mathbb{U}^{N}(x)$ to problem (3), the input $u^{0}(0)$ is applied to the system, before this procedure is repeated at the next time step $t+1$.
Now given the system dynamics $f$, the stage cost function $\ell$ and the constraint set $\mathbb{Y}$, it was shown that dissipativity with respect to the supply rate $s(x, u)=\ell(x, u)-\ell\left(x^{e}, u^{e}\right)$ is both sufficient [1] and (under a suitable controllability condition) necessary [6] for optimal steady-state operation at $x^{e}$, which means that no state and input sequence pair leads to an average performance better than the steady-state $\operatorname{cost} \ell\left(x^{e}, u^{e}\right)$, i.e., $\liminf _{N \rightarrow \infty}(1 / N) J_{N}\left(x_{0}, u\right) \geq \ell\left(x^{e}, u^{e}\right)$ for all $x_{0} \in \mathbb{X}$ and all $u \in \mathbb{U}^{\infty}\left(x_{0}\right)$. Furthermore, strict dissipativity with respect to the supply rate $s(x, u)=\ell(x, u)-$ $\ell\left(x^{e}, u^{e}\right)$ and bounded storage function together with suitable controllability conditions is sufficient [7] and necessary [8] for the so-called turnpike property of (near) optimal solutions of problem (3). This property means that (near) optimal solutions of problem (3) most of the time stay in a neighborhood of the equilibrium $x^{e}$, which can be employed to conclude (near) optimal performance and convergence to the steady-state $x^{e}$ for the closed-loop system resulting from the above economic MPC scheme [11]. Similar closed-loop convergence and performance results based on the above strict dissipativity property are also available for economic MPC schemes with additional terminal constraints $[1,20]$. For a more detailed discussion on the role of dissipativity in economic MPC, we also refer to [4].

As motivated in the Introduction, in many applications from economics optimal control problems with discounted stage cost are of interest, i.e., problems of the form

$\min _{u \in \mathbb{U}^{N}(x)} J_{N}(x, u)$ with $J_{N}(x, u)=\sum_{k=0}^{N-1} \beta^{k} \ell\left(x_{u}(k, x), u(k)\right)$,

with discount factor $0<\beta<1$. In order to establish similar relations between (strict) dissipativity, optimal steady-state operation and closed-loop performance and convergence as in the undiscounted case, a recent paper [12] proposed a notion of discounted dissipativity, which is as follows.

Definition 3: Given an equilibrium $\left(x^{e}, u^{e}\right)$ and a discount factor $0<\beta<1$, the system (1) is discounted strictly dissipative with respect to the supply rate $s: \mathbb{Y} \rightarrow \mathbb{R}$ if there exists a storage function $\lambda: \mathbb{R}^{n} \rightarrow \mathbb{R}$ bounded from below with $\lambda\left(x^{e}\right)=0$ and a function $\alpha \in \mathcal{K}_{\infty}$ such that the following inequality is satisfied for all $(x, u) \in \mathbb{Y}$ :

$$
\beta \lambda(f(x, u))-\lambda(x) \leq s(x, u)-\alpha\left(\left|\left(x-x^{e}, u-u^{e}\right)\right|\right) .
$$

Note that the requirement $\lambda\left(x^{e}\right)=0$ is crucial for the following statements and cannot be trivially satisfied as in the undiscounted case. ${ }^{1}$ As shown in [12], discounted strict dissipativity with respect to the supply rate $s(x, u)=$ $\ell(x, u)-\ell\left(x^{e}, u^{e}\right)$ implies that $\left(x^{e}, u^{e}\right)$ is an optimal equilibrium in the sense that

$$
V_{\infty}\left(x^{e}\right):=\min _{u \in \mathbb{U}^{\infty}\left(x^{e}\right)} J_{\infty}\left(x^{e}, u\right)=\ell\left(x^{e}, u^{e}\right) /(1-\beta)
$$

\footnotetext{
${ }^{1}$ In the undiscounted case, $\lambda+c$ for all $c \in \mathbb{R}$ is a storage function if $\lambda$ is one, which is not the case anymore in the discounted setting.
} 
which means that for the initial condition $x_{0}=x^{e}$, the constant input $u^{e}$ is optimal. Furthermore, this strict dissipativity condition can again be employed to conclude a certain turnpike property of optimal solutions to problem (4), which in turn can again be used to establish closed-loop performance and convergence guarantees for discounted economic MPC [13].

In summary, in both the standard (undiscounted) as well as the discounted case, a certain dissipativity condition has turned out to play a crucial role in the study of optimal control and economic MPC problems. Here, the undiscounted and the discounted strict dissipativity notions are compatible in the sense that for $\beta=1$ the discounted condition becomes the undiscounted one. In the following, we consider the question whether strict dissipativity implies discounted strict dissipativity for discount factors $\beta$ sufficiently close to one. Considering the above, a positive answer would imply that steady-state optimality, turnpike properties and closed-loop performance and convergence statements in economic MPC are preserved for a "mild" enough discounting. This would be in analogy to the stabilizing case (with positive definite cost function $\ell$ ), where asymptotic stability of the optimally controlled system is preserved for discount factors $\beta$ close enough to one (compare [21]). While the answer to the above question is negative in the most general case (compare the example in Section IV), under certain regularity conditions it is indeed true, as shown in the following section.

\section{DISSIPATIVITY AND DISCOUNTED DISSIPATIVITY}

In this section, we show under what conditions strict dissipativity implies discounted strict dissipativity for discount factors $\beta$ sufficiently close to one. To this end, we briefly recall some results from nonlinear programming. Namely, consider an optimization problem of the form $\min _{h^{0}(y)=0, g^{0}(y) \leq 0} f^{0}(y)$, where $y \in \mathbb{R}^{n_{y}}$ and the functions $f^{0}: \mathbb{R}^{n_{y}} \rightarrow \mathbb{R}, h^{0}: \mathbb{R}^{n_{y}} \rightarrow \mathbb{R}^{n_{h}}$ and $g^{0}: \mathbb{R}^{n_{y}} \rightarrow \mathbb{R}^{n_{g}}$ are twice continuously differentiable. Denote the set of active inequality constraints at a feasible point $y$ by $A(y):=$ $\left\{1 \leq j \leq n_{g}: g_{j}^{0}(y)=0\right\}$. A feasible point $y$ is regular if $\nabla_{y} h_{i}^{0}(y), 1 \leq i \leq n_{h}$, and $\nabla_{y} g_{j}^{0}(y), j \in A(y)$, are linearly independent. If a point $y^{*}$ is regular and a local minimizer of the above optimization problem, there exist (unique) Lagrange multiplier vectors $\nu \in \mathbb{R}^{n_{h}}$ and $\mu \in \mathbb{R}_{\geq 0}^{n_{g}}$ such that $\nabla_{y} f^{0}\left(y^{*}\right)+\nu^{T} \nabla_{y} h^{0}\left(y^{*}\right)+\mu^{T} \nabla_{y} g^{0}\left(y^{*}\right)=0$ with $\mu_{j}=0$ for all $j \notin A\left(y^{*}\right)$, see, e.g., [22, Proposition 3.3.1]. Furthermore, in the following we will make use of the second order sufficiency conditions [22, Proposition 3.3.2], i.e. (i) $w^{T} \nabla_{y}^{2}\left(f^{0}\left(y^{*}\right)+\nu^{T} h^{0}\left(y^{*}\right)+\mu^{T} g^{0}\left(y^{*}\right)\right) w>0$ for all $w \neq 0$ such that $\nabla_{y} h^{0}\left(y^{*}\right) w=0$ and $\nabla_{y} g_{j}^{0}\left(y^{*}\right) w=0$ for all $j \in A\left(y^{*}\right)$, and ${ }^{2}$ (ii) $\mu_{j}>0$ for all $j \in A\left(y^{*}\right)$.

\section{A. Optimal control related supply rates}

In the following, we assume that the state and input constraint set $\mathbb{Y}$ is given in terms of inequality constraints,

\footnotetext{
${ }^{2}$ Condition (ii) is typically called strict complementarity condition.
}

i.e., $\mathbb{Y}=\left\{(x, u) \in \mathbb{R}^{n} \times \mathbb{R}^{m}: g(x, u) \leq 0\right\}$ for some $g: \mathbb{R}^{n} \times \mathbb{R}^{m} \rightarrow \mathbb{R}^{p}$. Now consider the optimization problem

$$
\min _{x=f(x, u), g(x, u) \leq 0} \ell(x, u) .
$$

Clearly, if system (1) is (strictly) dissipative with respect to the supply rate $s(x, u)=\ell(x, u)-\ell\left(x^{e}, u^{e}\right)$ for some equilibrium $\left(x^{e}, u^{e}\right)$, then this equilibrium is a minimizer of problem (7). Now consider the function

$$
\tilde{\ell}(x, u):=\ell(x, u)-\ell\left(x^{e}, u^{e}\right)+\lambda(x)-\lambda(f(x, u)) .
$$

If the system is strictly dissipative with respect to the supply rate $s(x, u)=\ell(x, u)-\ell\left(x^{e}, u^{e}\right)$, from (2) it follows that $\tilde{\ell}(x, u) \geq \alpha\left(\left|\left(x-x^{e}, u-u^{e}\right)\right|\right)$ for all $(x, u) \in \mathbb{Y}$. This means that

$$
0=\tilde{\ell}\left(x^{e}, u^{e}\right)=\min _{g(x, u) \leq 0} \tilde{\ell}(x, u),
$$

i.e., $\left(x^{e}, u^{e}\right)$ is the unique minimizer of $\tilde{\ell}$ on the set $\mathbb{Y}$. We now impose the following assumption.

Assumption 4: (i) The functions $f, \ell$, and $g$, are twice continuously differentiable.

(ii) The point $\left(x^{e}, u^{e}\right)$ is a regular point of problem (7) and satisfies the second order sufficiency conditions.

(iii) The system is strictly dissipative with respect to the supply rate $s(x, u)=\ell(x, u)-\ell\left(x^{e}, u^{e}\right)$. Furthermore, the storage function $\lambda$ is twice continuously differentiable and $\left(x^{e}, u^{e}\right)$ satisfies the second order sufficiency conditions for problem (9).

We are now in a position to prove the following result.

Theorem 5: Let Assumption 4 be satisfied. Then there exists $\hat{\beta}$ such that for all $\hat{\beta} \leq \beta \leq 1$, there exists an equilibrium $\left(x^{e}(\beta), u^{e}(\beta)\right)$ such that the system is discounted strictly dissipative with respect to the supply rate $s(x, u)=$ $\ell(x, u)-\ell\left(x^{e}(\beta), u^{e}(\beta)\right)$, i.e., there exist a storage function $\hat{\lambda}(x, \beta)$ with $\hat{\lambda}\left(x^{e}(\beta), \beta\right)=0$ and $\sigma \in \mathcal{K} \mathcal{L}$ such that the function

$$
\begin{aligned}
\hat{\ell}(x, u, \beta):= & \ell(x, u)-\ell\left(x^{e}(\beta), u^{e}(\beta)\right) \\
& +\hat{\lambda}(x, \beta)-\beta \hat{\lambda}(f(x, u), \beta),
\end{aligned}
$$

satisfies $\hat{\ell}(x, u, \beta) \geq \sigma\left(\left|\left(x-x^{e}(\beta), u-u^{e}(\beta)\right)\right|\right)$ for all $(x, u) \in \mathbb{Y}$.

Proof: Let $h(x, u, \beta):=x-\beta f(x, u)$ and consider the set of equations

$$
\begin{aligned}
& \nabla_{(x, u)} \ell(x, u)+\nu^{T} \nabla_{(x, u)} h(x, u, \beta)+\mu^{T} \nabla_{(x, u)} g(x, u)=0 \\
& x-f(x, u)=0, \\
& g_{i}(x, u)+z_{i}^{2}=0, \quad i=1, \ldots, p \\
& 2 \mu_{i} z_{i}=0, \quad i=1, \ldots, p
\end{aligned}
$$

where $\nu \in \mathbb{R}^{n}, \mu \in \mathbb{R}^{p}$, and $z \in \mathbb{R}^{p}$. For each fixed $\beta$, (11) is a set of $2 n+m+2 p$ equations for $2 n+m+2 p$ unknowns $x, u, \nu, \mu, z$. Since $\left(x^{e}, u^{e}\right)$ is regular and a minimizer of problem (7), for $\beta=1$ it follows that $x=x^{e}, u=u^{e}$, and $z_{i}=\sqrt{-g_{i}\left(x^{e}, u^{e}\right)}=: z_{i}^{e}$ together with some (unique) $\nu=\nu^{e}$ and $\mu=\mu^{e} \geq 0$ are a solution to (11), since for 
these values the set of equations (11) corresponds to the Karush-Kuhn-Tucker (KKT) conditions of problem (7) (see, e.g., [22, Proposition 3.1.1]). The corresponding Jacobian $J$ of (11) with respect to $(x, u, \nu, \mu, z)$ is given by

$$
J=\left[\begin{array}{cccc}
A & b^{T} & c^{T} & 0 \\
b & 0 & 0 & 0 \\
c & 0 & 0 & 2 \operatorname{diag}\left(z^{e}\right) \\
0 & 0 & 2 \operatorname{diag}\left(z^{e}\right) & 2 \operatorname{diag}\left(\mu^{e}\right)
\end{array}\right]
$$

where $A:=\nabla_{(x, u)}^{2} \ell\left(x^{e}, u^{e}\right)+\sum_{i=1}^{n} \nu_{i}^{e} \nabla_{(x, u)}^{2} h_{i}\left(x^{e}, u^{e}, 1\right)+$ $\sum_{i=1}^{p} \mu_{i}^{e} \nabla_{(x, u)}^{2} g_{i}\left(x^{e}, u^{e}, 1\right), b=\nabla_{(x, u)} h\left(x^{e}, u^{e}, 1\right)$, and $c=$ $\nabla_{(x, u)} g\left(x^{e}, u^{e}\right)$. Since by property (ii) of Assumption 4, the second order sufficiency conditions for problem (7) are satisfied, it follows that $J$ is nonsingular (compare [22, Section 3.3.3]). Hence we can use the implicit function theorem to conclude that for $\beta$ sufficiently close to one, there exists a solution $x^{e}(\beta), u^{e}(\beta), z(\beta), \nu(\beta), \mu(\beta)$ to (11) such that the functions $x^{e}(\cdot), u^{e}(\cdot), z(\cdot), \nu(\cdot), \mu(\cdot)$ are continuously differentiable and $x^{e}(1)=x^{e}, u^{e}(1)=u^{e}, z(1)=z^{e}$, $\nu(1)=\nu^{e}$, and $\mu(1)=\mu^{e}$. Furthermore, from continuity of $\mu(\cdot)$ and $z(\cdot)$, the fourth equation of (11), and the fact that $\mu_{i}^{e}>0$ for all $i \in A\left(x^{e}, u^{e}\right)$ by Assumption 4 (iii), it follows that for $\beta$ sufficiently close to one, $\mu(\beta)>0$ if $\mu^{e}>0$, $\mu(\beta)=0$ if $\mu^{e}=0$, and $A\left(x^{e}(\beta), u^{e}(\beta)\right)=A\left(x^{e}, u^{e}\right)$.

Next, since by Assumption 4 (ii) and (iii), $\left(x^{e}, u^{e}\right)$ is a regular point of problem (7) (and hence also of problem (9)) and $\left(x^{e}, u^{e}\right)$ is a strict minimizer of $\tilde{\ell}$ on the set $\mathbb{Y}$, it follows that the KKT conditions $\nabla_{(x, u)} \tilde{\ell}\left(x^{e}, u^{e}\right)+$ $\tilde{\mu}^{T} g\left(x^{e}, u^{e}\right)=0$ are satisfied for some $\tilde{\mu} \in \mathbb{R}_{\geq_{0}}^{p}$ [22, Proposition 3.1.1]. But then, since $\nabla_{(x, u)}\left(\lambda\left(x^{e}\right)-\lambda\left(f\left(x^{e}, u^{e}\right)\right)\right)=$ $\nabla_{x} \lambda\left(x^{e}\right) \nabla_{(x, u)} h\left(x^{e}, u^{e}, 1\right)$, from (11) with $\beta=1$ and uniqueness of the Lagrange multiplier vectors $\nu^{e}$ and $\mu^{e}$ it follows that $\nabla_{x} \lambda\left(x^{e}\right)=\left(\nu^{e}\right)^{T}$ and $\tilde{\mu}=\mu^{e}$.

Now define

$$
\begin{aligned}
\hat{\lambda}(x, \beta):= & \lambda(x)-\lambda\left(x^{e}(\beta)\right) \\
& +\left(\nu(\beta)^{T}-\nabla_{x} \lambda\left(x^{e}(\beta)\right)\right)\left(x-x^{e}(\beta)\right) .
\end{aligned}
$$

First, note that $\hat{\lambda}\left(x^{e}(\beta), \beta\right)=0$. We now want to show that for $\beta$ sufficiently close to one, $\left(x^{e}(\beta), u^{e}(\beta)\right)$ is a (local) minimizer of $\hat{\ell}$ as defined in (10), i.e., for the optimization problem

$$
\min _{g(x, u) \leq 0} \hat{\ell}(x, u, \beta) .
$$

To this end, we show that the KKT conditions and the second order sufficiency conditions for this problem are satisfied. Since $\nabla_{x} \hat{\lambda}\left(x^{e}(\beta), \beta\right)=\nu(\beta)^{T}$, we obtain

$$
\begin{aligned}
& \nabla_{(x, u)} \hat{\ell}\left(x^{e}(\beta), u^{e}(\beta), \beta\right) \\
& =\nabla_{(x, u)} \ell\left(x^{e}(\beta), u^{e}(\beta)\right) \\
& \quad+\nabla_{(x, u)}\left(\hat{\lambda}\left(x^{e}(\beta), \beta\right)-\beta \hat{\lambda}\left(f\left(x^{e}(\beta), u^{e}(\beta)\right), \beta\right)\right) \\
& =\nabla_{(x, u)} \ell\left(x^{e}(\beta), u^{e}(\beta)\right) \\
& \quad+\nabla_{x} \hat{\lambda}\left(x^{e}(\beta), \beta\right) \nabla_{(x, u)} h\left(x^{e}(\beta), u^{e}(\beta), \beta\right) \\
& =\nabla_{(x, u)} \ell\left(x^{e}(\beta), u^{e}(\beta)\right)+\nu(\beta)^{T} \nabla_{(x, u)} h\left(x^{e}(\beta), u^{e}(\beta), \beta\right) .
\end{aligned}
$$

Combining this with the above established fact that $x=$ $x^{e}(\beta), u=u^{e}(\beta), \nu=\nu(\beta), \mu=\mu(\beta)$ satisfy the first equation of (11) results in $\nabla_{(x, u)} \hat{\ell}\left(x^{e}(\beta), u^{e}(\beta), \beta\right)+$ $\mu(\beta)^{T} \nabla_{(x, u)} g\left(x^{e}(\beta), u^{e}(\beta)\right)=0$. Together with the fact that $\mu(\beta) \geq 0$ and $\mu_{i}(\beta)=0$ for all $i \notin A\left(x^{e}(\beta), u^{e}(\beta)\right)$, this means that the KKT conditions for problem (14) are satisfied at $\left(x^{e}(\beta), u^{e}(\beta)\right)$. Next, since $x^{e}(\cdot), u^{e}(\cdot)$, and $\nu(\cdot)$ are continuous and $\nabla_{x} \lambda\left(x^{e}\right)=\left(\nu^{e}\right)^{T}$ as discussed above, it follows that $\nabla_{(x, u)}^{2} \hat{\ell}\left(x^{e}(\cdot), u^{e}(\cdot), \cdot\right)$ is continuous with $\nabla_{(x, u)}^{2} \hat{\ell}\left(x^{e}(1), u^{e}(1), 1\right)=\nabla_{(x, u)}^{2} \tilde{\ell}\left(x^{e}, u^{e}\right)$. But then, since the second order sufficiency conditions for problem (9) are satisfied by Assumption 4 (iii), i.e., (i) $y^{T} \nabla_{(x, u)}^{2}\left(\tilde{\ell}\left(x^{e}, u^{e}\right)+\left(\mu^{e}\right)^{T} g\left(x^{e}, u^{e}\right)\right) y>0$ for all $y \neq 0$ such that $\nabla_{(x, u)} g_{i}\left(x^{e}, u^{e}\right) y=0$ for all $i \in A\left(x^{e}, u^{e}\right)$ and (ii) $\mu_{i}^{e}>0$ for all $i \in A\left(x^{e}, u^{e}\right)$, by continuity and the fact that $A\left(x^{e}(\beta), u^{e}(\beta)\right)=A\left(x^{e}, u^{e}\right)$ it follows that also the second order sufficiency conditions for problem (14) are satisfied, i.e., (i) $y^{T} \nabla_{(x, u)}^{2}\left(\hat{\ell}\left(x^{e}(\beta), u^{e}(\beta)\right)+\right.$ $\left.\mu(\beta)^{T} g\left(x^{e}(\beta), u^{e}(\beta)\right)\right) y>0$ for all $y \neq 0$ such that $\nabla_{(x, u)} g_{i}\left(x^{e}(\beta), u^{e}(\beta)\right) y=0$ for all $i \in A\left(x^{e}(\beta), u^{e}(\beta)\right)$ and (ii) $\mu_{i}(\beta)>0$ for all $i \in A\left(x^{e}(\beta), u^{e}(\beta)\right)$. Hence for $\beta$ sufficiently close to one, $\left(x^{e}(\beta), u^{e}(\beta)\right)$ is a strict local minimizer of $\hat{\ell}$ (see, e.g. [22, Proposition 3.3.2]). But then, since $\left(x^{e}, u^{e}\right)$ was a global minimizer of $\tilde{\ell}$ on the compact set $\mathbb{Y}$, by continuity $\beta$ can be chosen close enough to one such that also $\left(x^{e}(\beta), u^{e}(\beta)\right)$ is a global minimizer of $\hat{\ell}$ on $\mathbb{Y}$, i.e., there exists $\sigma \in \mathcal{K} \mathcal{L}$ such that $\hat{\ell}(x, u, \beta) \geq$ $\sigma\left(\left|\left(x-x^{e}(\beta), u-u^{e}(\beta)\right)\right|\right)$ for all $(x, u) \in \mathbb{Y}$. Together with the fact that $\hat{\lambda}\left(x^{e}(\beta), \beta\right)=0$ as established above, this implies that the system is discounted strictly dissipative with respect to the supply rate $s(x, u)=\ell(x, u)-\ell\left(x^{e}(\beta), u^{e}(\beta)\right)$, which concludes the proof of Theorem 5 .

Remark 6: In [6, Theorem 5], robustness of (undiscounted) dissipativity with respect to parameter variations in the constraint set $\mathbb{Y}$ was studied. Both the above proof of Theorem 5 and the proof of [6, Theorem 5] use ideas from the context of nonlinear programming. However, while in $[6$, Theorem 5] one could directly apply sensitivity results, this was not the case in the above proof, since for $\beta \neq 1$, the set of equations (11) do not correspond to the KKT conditions of some associated optimization problem, but only for $\beta=1$.

\section{B. Extension to general supply rates}

We now briefly discuss how the preceding results can be extended to general supply rates. Namely, given an equilibrium $\left(x^{e}, u^{e}\right)$, suppose that system (1) is strictly dissipative with respect to some supply rate $s: \mathbb{Y} \rightarrow \mathbb{R}$. We can now distinguish two cases. First, if the minimum value of the problem

$$
\min _{g(x, u) \leq 0} s(x, u)+\lambda(x)-\lambda(f(x, u))
$$

is (strictly) positive and $f, s$, and $\lambda$ are continuous, then also $\min _{g(x, u) \leq 0} s(x, u)+\lambda(x)-\beta \lambda(f(x, u))>0$ for $\beta$ close enough to one (due to compactness of $\mathbb{Y}$ ). Hence system (1) is also discounted strictly dissipative in this case. Second, if the minimum value of the problem (15) is zero, by strict 


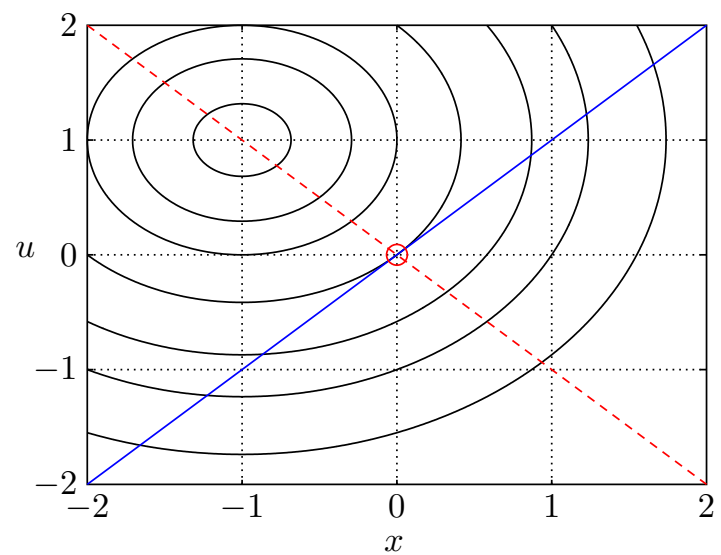

Fig. 1. Illustration of the steady-states (blue solid line), level sets of $\ell$ (black ellipses), and the additional constraint $g_{a d}$ (red dashed) of the example in Section IV. The optimal steady-state $\left(x^{e}, u^{e}\right)=(0,0)$ for the undiscounted case is marked with a circle.

dissipativity it follows that the minimizer of problem (15) is the point $\left(x^{e}, u^{e}\right)$, which is also the minimizer to the problem

$$
\min _{x=f(x, u), g(x, u) \leq 0} s(x, u) .
$$

In this case, discounted strict dissipativity can be shown analogously to the proof of Theorem 5, using the following modified assumption.

Assumption 7: (i) The functions $f, s, g$, and $\lambda$ are twice continuously differentiable.

(ii) The point $\left(x^{e}, u^{e}\right)$ is a regular point and satisfies the second order sufficiency conditions of problems (15) and (16).

We then arrive at the following corollary.

Corollary 8: Suppose that system (1) is strictly dissipative with respect to the supply rate $s$ and that either (i) the minimum value of the problem (15) is positive and $f, s$, and $\lambda$ are continuous, or (ii) Assumption 7 holds. Then there exists $\hat{\beta}$ such that for all $\hat{\beta} \leq \beta \leq 1$, the system is discounted strictly dissipative with respect to the supply rate $s$.

\section{EXAMPLE}

We now illustrate the preceding results with a simple example. Consider the system

$$
x(k+1)=u(k)
$$

with stage cost $\ell(x, u)=(x+1)^{2}+(u-1)^{2}$ and state and input constraints given by

$$
g(x, u)=\left[\begin{array}{c}
-2-x \\
x-2 \\
-2-u \\
u-2
\end{array}\right] .
$$

The optimal equilibrium is $\left(x^{e}, u^{e}\right)=(0,0)$ with associated stage cost $\ell\left(x^{e}, u^{e}\right)=2$. One can show that the system (17) is strictly dissipative with respect to the supply rate $s(x, u)=$ $\ell(x, u)-\ell\left(x^{e}, u^{e}\right)$ and storage function $\lambda(x)=-2 x$. The point $\left(x^{e}, u^{e}\right)=(0,0)$ is a regular point of problem (7) and satisfies the second order sufficiency conditions for problems (7) and (9) (note that both $\ell$ and $\tilde{\ell}$ are quadratic and none of the constraints specified by $g$ are active at $\left.\left(x^{e}, u^{e}\right)=(0,0)\right)$. Hence Assumption 4 is satisfied and we can apply Theorem 5 to conclude that there exists an equilibrium $\left(x^{e}(\beta), u^{e}(\beta)\right)$ such that the system (17) is also discounted strictly dissipative with respect to the supply rate $s(x, u)=\ell(x, u)-\ell\left(x^{e}(\beta), u^{e}(\beta)\right)$ for discount factors $\beta$ close enough to one ${ }^{3}$. Indeed, as shown in the proof of Theorem 5, the optimal equilibrium $\left(x^{e}(\beta), u^{e}(\beta)\right)$ varies continuously in $\beta$ and is given by

$$
\left(x^{e}(\beta), u^{e}(\beta)\right)=\left(\frac{1-\beta}{1+\beta}, \frac{1-\beta}{1+\beta}\right) .
$$

The corresponding storage function $\hat{\lambda}$ is given by

$$
\hat{\lambda}(x, \beta)=-\frac{4}{1+\beta}\left(x-\frac{1-\beta}{1+\beta}\right),
$$

which is in accordance with (13).

Now consider the same example but with additional constraint $g_{a d}(x, u)=x+u \leq 0$, i.e., the state and input constraint set $\mathbb{Y}$ is determined by

$$
g(x, u)=\left[\begin{array}{c}
-2-x \\
x-2 \\
-2-u \\
u-2 \\
x+u
\end{array}\right]
$$

Since $\left(x^{e}, u^{e}\right)=(0,0)$ is still a feasible point (however, now on the boundary of the set $\mathbb{Y}$ ), clearly the system (17) is still strictly dissipative with respect to the supply rate $s(x, u)=$ $\ell(x, u)-\ell\left(x^{e}, u^{e}\right)$ and storage function $\lambda(x)=-2 x$ as above. On the other hand, for any $0<\beta<1$, the equilibrium $\left(x^{e}(\beta), u^{e}(\beta)\right)$ given by (18) is not feasible anymore. Indeed, for any $0<\beta<1$, the system is not discounted strictly dissipative anymore. According to the results in Section II$\mathrm{B}$, this can be proven by showing that none of the feasible equilibria is optimal, i.e., for all feasible equilibria $\left(x^{e}, u^{e}\right)$ we have $V_{\infty}\left(x^{e}\right)<\ell\left(x^{e}, u^{e}\right) /(1+\beta)$. Namely, given any feasible equilibrium $-2 \leq x^{e} \leq 0$ with $u^{e}=x^{e}$, consider the input and corresponding state sequences $u^{\prime}=$ $(\delta,-\delta, \delta, \ldots)$ and $x^{\prime}=\left(x^{e}, \delta,-\delta, \delta, \ldots\right)$ for some $\delta>0$. Straightforward (but cumbersome) computations show that $J_{\infty}\left(x^{e}, u^{\prime}\right)<\ell\left(x^{e}, u^{e}\right) /(1+\beta)$ if $\delta<2(1-\beta)^{2} /(1+\beta)^{2}$. Hence for each $0<\beta<1$, there exists some $\delta>0$ such that $V_{\infty}\left(x^{e}\right) \leq J_{\infty}\left(x^{e}, u^{\prime}\right)<\ell\left(x^{e}, u^{e}\right) /(1+\beta)$. This means that for each $0<\beta<1$, the system is not discounted strictly dissipative. The reason why Theorem 5 fails is that the second order sufficiency conditions for problems (7) and (9) are not satisfied (while the rest of Assumption 4 is true). Namely, the strict complementarity condition is not satisfied, since the constraint $x+u \leq 0$ is active at $\left(x^{e}, u^{e}\right)=(0,0)$, but the corresponding Lagrange multiplier is zero.

\footnotetext{
${ }^{3}$ In fact, since the system is linear with strictly convex stage cost $\ell$, it is discounted strictly dissipative for all $0<\beta<1$, compare [12, Theorem 6].
} 


\section{Conclusions}

In conclusion, we have shown that under certain regularity conditions known from the context of nonlinear programming, strict dissipativity implies discounted strict dissipativity for discount factors close enough to one. This was first shown for a supply rate which is of interest in the context of (discounted) optimal control and economic MPC, and was subsequently extended to general supply rates $s$. The presented results allow to conclude that steady-state optimality, turnpike properties and closed-loop performance and convergence statements in economic MPC are preserved under a mild enough discounting.

\section{REFERENCES}

[1] D. Angeli, R. Amrit, and J. B. Rawlings, "On average performance and stability of economic model predictive control," IEEE Transactions on Automatic Control, vol. 57, no. 7, pp. 1615-1626, 2012.

[2] M. Ellis, H. Durand, and P. D. Christofides, "A tutorial review of economic model predictive control methods," Journal of Process Control, vol. 24, no. 8, pp. 1156 - 1178, 2014.

[3] M. A. Müller and F. Allgöwer, "Economic and distributed model predictive control: recent developments in optimization-based control," SICE Journal of Control, Measurement, and System Integration, vol. 10, no. 2, pp. 1-14, 2017.

[4] M. A. Müller, L. Grüne, and F. Allgöwer, "On the role of dissipativity in economic model predictive control," in Proceedings of the 5th IFAC Conference on Nonlinear Model Predictive Control, 2015, pp. 110116.

[5] J. C. Willems, "Dissipative dynamical systems - part i: General theory," Archive for Rational Mechanics and Analysis, vol. 45, no. 5, pp. 321351, 1972.

[6] M. A. Müller, D. Angeli, and F. Allgöwer, "On necessity and robustness of dissipativity in economic model predictive control," IEEE Transactions on Automatic Control, vol. 60, no. 6, pp. 1671-1676, 2015.

[7] L. Grüne, "Economic receding horizon control without terminal constraints," Automatica, vol. 49, no. 3, pp. 725-734, 2013.

[8] L. Grüne and M. A. Müller, "On the relation between strict dissipativity and turnpike properties," System \& Control Letters, vol. 90, pp. 45-53, 2016.

[9] T. Faulwasser, M. Korda, C. N. Jones, and D. Bonvin, "Turnpike and dissipativity properties in dynamic real-time optimization and economic MPC," in Proceedings of the 53rd IEEE Conference on Decision and Control, 2014, pp. 2734-2739.

[10] R. Amrit, J. B. Rawlings, and D. Angeli, "Economic optimization using model predictive control with a terminal cost," Annual Reviews in Control, vol. 35, no. 2, pp. 178-186, 2011.

[11] L. Grüne and M. Stieler, "Asymptotic stability and transient optimality of economic MPC without terminal constraints," Journal of Process Control, vol. 24, no. 8, pp. 1187-1196, 2014.

[12] L. Grüne, C. M. Kellett, and S. R. Weller, "On a discounted notion of strict dissipativity," in Proceedings of the 10th IFAC Symposium on Nonlinear Control Systems, 2016, pp. 247-252.

[13] L. Grüne, W. Semmler, and M. Stieler, "Using nonlinear model predictive control for dynamic decision problems in economics," Journal of Economic Dynamics and Control, vol. 60, pp. 112 - 133, 2015.

[14] A. Seierstad and K. Sydsæter, Optimal Control Theory with Economic Applications. North-Holland, Amsterdam, 1987.

[15] W. Nordhaus, "Estimates of the social cost of carbon: Concepts and results from the DICE-2013R model and alternative approaches," $J$. Assoc. Environ. Resour. Econ., vol. 1, no. 1/2, pp. 273-312, Mar. 2014.

[16] S. R. Weller, S. Hafeez, and C. M. Kellett, "A receding horizon control approach to estimating the social cost of carbon in the presence of emissions and temperature uncertainty," in Proc. 54th IEEE Conf. Decis. Control, Osaka, Japan, 15-18 December 2015, pp. 5384-5390.

[17] C. I. Byrnes and W. Lin, "Losslessness, feedback equivalence, and the global stabilization of discrete-time nonlinear systems," IEEE Transactions on Automatic Control, vol. 39, no. 1, pp. 83-98, 1994.
[18] M. A. Müller and L. Grüne, "Economic model predictive control without terminal constraints for optimal periodic behavior," Automatica, vol. 70, pp. 128-139, 2016.

[19] T. Faulwasser and D. Bonvin, "On the design of economic NMPC based on approximate turnpike properties," in Proceedings of the 54th IEEE Conference on Decision and Control, 2015, pp. 4964-4970.

[20] L. Grüne and A. Panin, "On non-averaged performance of economic MPC with terminal conditions," in Proceedings of the 54th IEEE Conference on Decision and Control, Dec 2015, pp. 4332-4337.

[21] V. Gaitsgory, L. Grüne, and N. Thatcher, "Stabilization with discounted optimal control," Systems \& Control Letters, vol. 82, pp. 91 $-98,2015$.

[22] D. P. Bertsekas, Nonlinear Programming. Belmont, Massachusetts: Athena Scientific, 1995. 